\title{
Anal phyllodes tumor in a male patient: a unique case presentation and literature review
}

\author{
Szu-pei Ho ${ }^{1}$, Hui-hwa Tseng ${ }^{1 *}$, TM King ${ }^{2}$ and Philip-C Chow ${ }^{3}$
}

\begin{abstract}
Lesions of anogenital mammary-like glands are rare, and only 44 female cases have been reported. Herein, we describe a particularly rare case of phyllodes tumor of anogenital mammary-like glands in a 41-year-old male presenting anal bleeding. Papillectomy was performed. The excised tumor was circumscribed in shape, and after it was sliced into sections, it was noted that there were leaf-like slits on the surface of cut side. Under the microscope, the tumor was found to be biphasic, with a bland glandular epithelium and low-to-intermediate cellular stroma, which together created the leaf-like slits. Gynecomastoid hyperplasia was evident at the periphery. The epithelium showed immuno-activity for ER, PR(focal), AR, and GCDFP-15. The stromal cells showed positive staining for CD34 and vimentin. The morphology and immunophenotype were similar to benign phyllodes tumors of breast. To the best of our knowledge, this case report represents the first case of phyllodes tumor of anogenital mammary-like glands with gynecomastoid hyperplasia at the periphery in a male patient. To make a diagnosis, we had to differentiate this lesion from hidradenoma papilliferum of skin appendage, phyllodes tumor of ectopic prostatic tissue, and other tumors of anogenital mammary-like glands analogous to the breast tumor (e.g., fibroadenoma phyllodes, periductal stromal sarcoma, and spindle cell carcinoma). While gynecomastia of male breast is usually a result of hormone imbalance, our patient's tumor did not seem to be related to peripheral hormone status in the anogenital mammary-like glands. Nevertheless, because hormone imbalance has been strongly related to male breast cancer, hormone levels may need to be followed in male patients who have this rare malady.

Virtual slides: The virtual slide(s) for this article can be found here: http://www.diagnosticpathology.diagnomx.eu/ vs/1509145815899177
\end{abstract}

Keywords: Anogenital mammary-like glands, Ectopic breast tissue, Fibroepithelial neoplasm, Phyllodes tumor, Gynecomastia

\section{Background}

Ectopic breast tissue in the vulva was first identified by Hartung in 1872, and it has long been considered caudal remnants of the milk ridges, though mammary type tissue has been reported to be a normal constituent of the anogenital area (1991) [1]. Today such tissues in the anal area are called anogenital mammary-like glands (AGMLG). There have been reports of lesions arising in AGMLG that resemble breast neoplasms, including benign or malignant, epithelial and/or stromal neoplasms. Phyllodes tumor or other fibroepithelial tumors of AGMLG is extremely

\footnotetext{
* Correspondence: ssssnowkimo@gmail.com

'Department of Pathology and Laboratory Medicine, Kaohsiung Veterans General Hospital, Kaohsiung, Taiwan

Full list of author information is available at the end of the article
}

rare and has previously been found in females exclusively [2-4]. Herein, we present the first case of a male patient found to have low-grade phyllodes tumor of AGMLG. Gynecomastoid hyperplasia of AGMLG was also identified at periphery of tumor. Because all fibroadenomas of the male breast have been found with concurrent gynecomastia and patients with these lesions have clear hormone imbalances [5], we assumed that the phyllodes tumor or other fibroepithelial neoplasms of AGMLG with gynecomastoid hyperplasia at the periphery might also suggest possible hormone imbalance. However, peripheral estrogen/androgen ratio was found to be unchanged.

\section{Biomed Central}

(c) 2013 Ho et al.; licensee BioMed Central Ltd. This is an Open Access article distributed under the terms of the Creative Commons Attribution License (http://creativecommons.org/licenses/by/2.0), which permits unrestricted use, distribution, and reproduction in any medium, provided the original work is properly cited. 


\section{Case presentation Case report}

A 41-year-old Taiwanese male with a previous history of hypertension and major depressive disorder under control with medication came to the outpatient clinic of the Division of Gastroenterology complaining of recent anal bleeding. Physical examination and colon fiberoscopy revealed a subepithelial tumor at anal verge (Figure 1), for which he received a papillectomy.

\section{Materials and methods}

For pathology analysis, representative specimens were fixed in $4 \%$ buffered formalin and embedded in paraffin. They were sliced into serial sections (4um) and stained with hematoxylin-eosin. Immunohistochemistry stains were performed using Leica BOND-MAX. We performed immunostaining for: estrogen receptor (Clone 6F11); progesterone (Clone 16); androgen receptor (Clone AR27); gross cystic disease fluid protein 15 (Clone 23A3); vimentin (Clone SRL33); CD34 (Clone QBEnd/10); actin (Clone HHF35); smooth muscle actin(SMA) (Clone alpha sm-1); Ki-67(MIB-1) (Clone GM010); Cytokeratin 7(CK7) (CloneOV-TL 12/30); Prostatic Acid Phosphatase (PSAP) (Clone PASE/4LJ); prostatespecific antigen (PSA) (Clone 35H9); Pan-cytokeratin (Clone AE1/AE3).

\section{Results}

Grossly, the subepithelial tumor measured $2.4 \mathrm{~cm}$ at the greatest circumference. It was grey-white, mildly firm, well-circumscribed with a cleft-like appearance (Figure 2). The skin overlying the anal tumor had no remarkable findings.

Microscopically, the circumscribed tumor was composed of the biphasic components of glandular epithelium

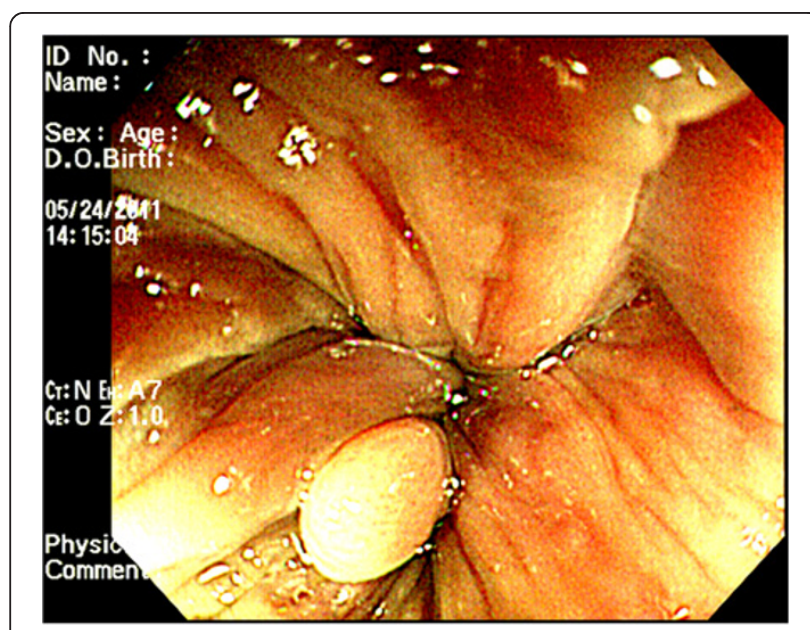

Figure 1 Colofibroscopic finding. The endoscopic findings revealed a subepithelial tumor at anal verge.

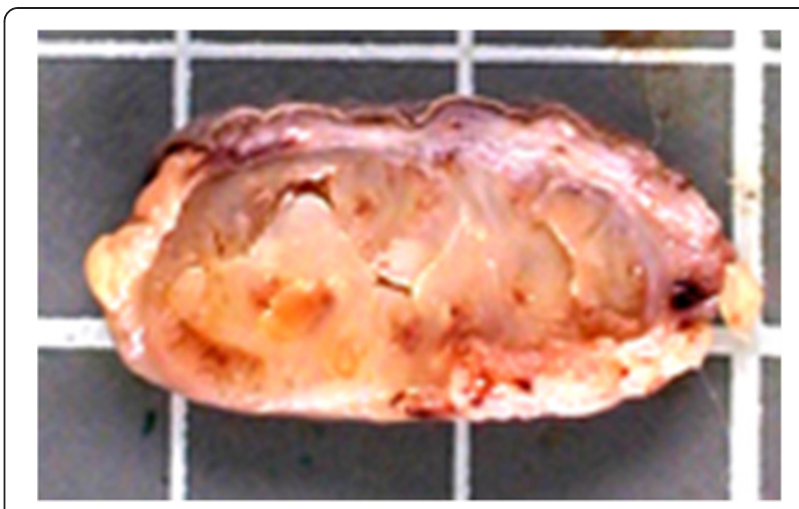

Figure 2 Gross features. The tumor appeared circumscribed. The cut sections showed leaf-like slits.

and stromal component, together forming leaf-like slits (Figure 3a). The glandular epithelium consisted of a luminal ductal epithelium layer and a basal myoepithelial layer. Stroma showed low-to-intermediate cellularity (Figure 3d-e). An increase in Ki-67 proliferative index was found in stromal cells. (Figure 4f). The morphology of the tumor was similar to that of the mammary glands under phyllodes change. At the peripheral of tumor, both ductal epithelium and periductal stroma proliferated without lobular pattern (Figure 3f). The stroma were myxoid or edematous in appearance. These characteristics were similar to those of gynecomastia on the male breast [5].

In the immunohistochemical study, the luminal ductal epithelium showed positive for ER and PR and focal positive for AR and GCDFP-15 (Figure 4a-d). The basal myoepithelial cells showed positive for CK5/6, actin, and SMA. The stromal cells showed positive for CD34 (Figure 4e) and vimentin and negative for actin and SMA.

The patient remained in the hospital for three days and recovery was unremarkable. He was discharged on day 4. As of 20 months post-surgery, there was no local recurrence.

\section{Discussion}

Lesions of AGMLG present as a spectra of morphologic changes synonymous with those in the breast, including lactating adenoma, hidradenoma papilliferum, syringoadenoma papilliferum, fibroadenoma, phyllodes tumor, pseudoangiomatous stromal hyperplasia, extramammary Paget's disease and other malignancies arising from AGMLG [6]. Benign changes, such as apocrine, oxyphilic or squamous metaplasia, myoepithelial hyperplasia or clear cell metaplasia, lactationlike change and florid epithelial hyperplasia, have also occasionally been identified [6]. To date, fibroepithelial lesions of AGMLG have only been reported in 44 female cases. As far as we know, there has been 


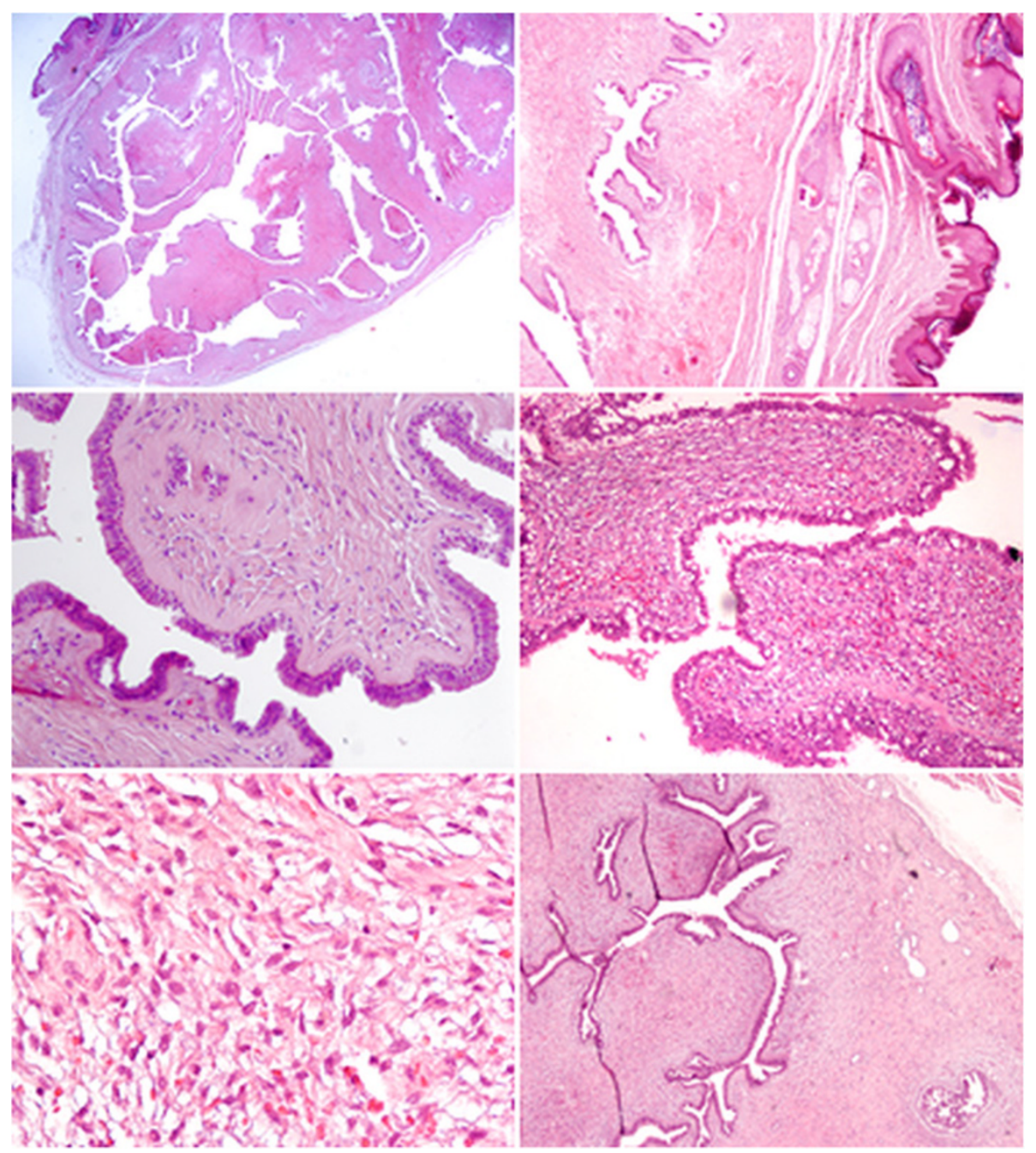

Figure 3 Microscopic findings. a) At the low power field, the tumor was found to be well-circumscribed. Leaf-like growth pattern was clearly depicted. b) The overlying skin and skin appendage of this tumor showed no remarkable change. c) The epithelium was composed of double layers. The stroma showed low cellularity. d) However, there is other area of tumor which shows increased stromal cellularity in low-to -intermediate degree. e) At high power field, stormal hypercellularity and cell atypism were identified. f) At peripheral, gynecomastoid hyperplasia featuring proliferation of both ductal and stromal cells was found.

no report of a case of this tumor of AGMLG in a male patient.

We performed differential diagnoses to exclude possible hidradenoma papilliferum of skin appendage, phyllodes tumor of ectopic prostatic tissue, and tumors of AGMLG which are analogous with the breast neoplasm. Hidradenoma papilliferum can also be found in the anal region [7-9]. However, hidradenoma papilliferum has a more complex papillary structure than the leaf-like pattern of benign phyllodes tumors [10]. In hidradenoma papilliferum, glandular secretion is incapacitated. Because lesions of mammary glands and sweat glands stain for ER and GCDFP-15, immunohistochemistry may not be used to distinguish hidradenoma papilliferum from mammary lesions [8]. Phyllodes tumor has been found in ectopic prostate tissue, mostly in female patients, the most common locations being the vulva, vagina, cervix, urinary bladder and anal canal [11-13]. Gynecomastoid hyperplasia around the main lesion may cause a pathologist to suspect residual normal prostate glands. Mammary glands may show weakly positive for PSA. However, a positive finding of CK7 and negative finding for PSAP may help exclude the possibility of a prostatic origin (Figure 4g-h) [11]. There are other tumors homologous to the breast tumor. One, periductal stromal sarcoma, also has a biphasic growth pattern. However, it does not have the leaf-like appearance of intracanicular growth pattern but rather a solid growth appearance of a pericanalicular pattern [14]. Another such tumor is spindle cell carcinoma. The epithelioid nests of this tumor may merge with spindle stromal 


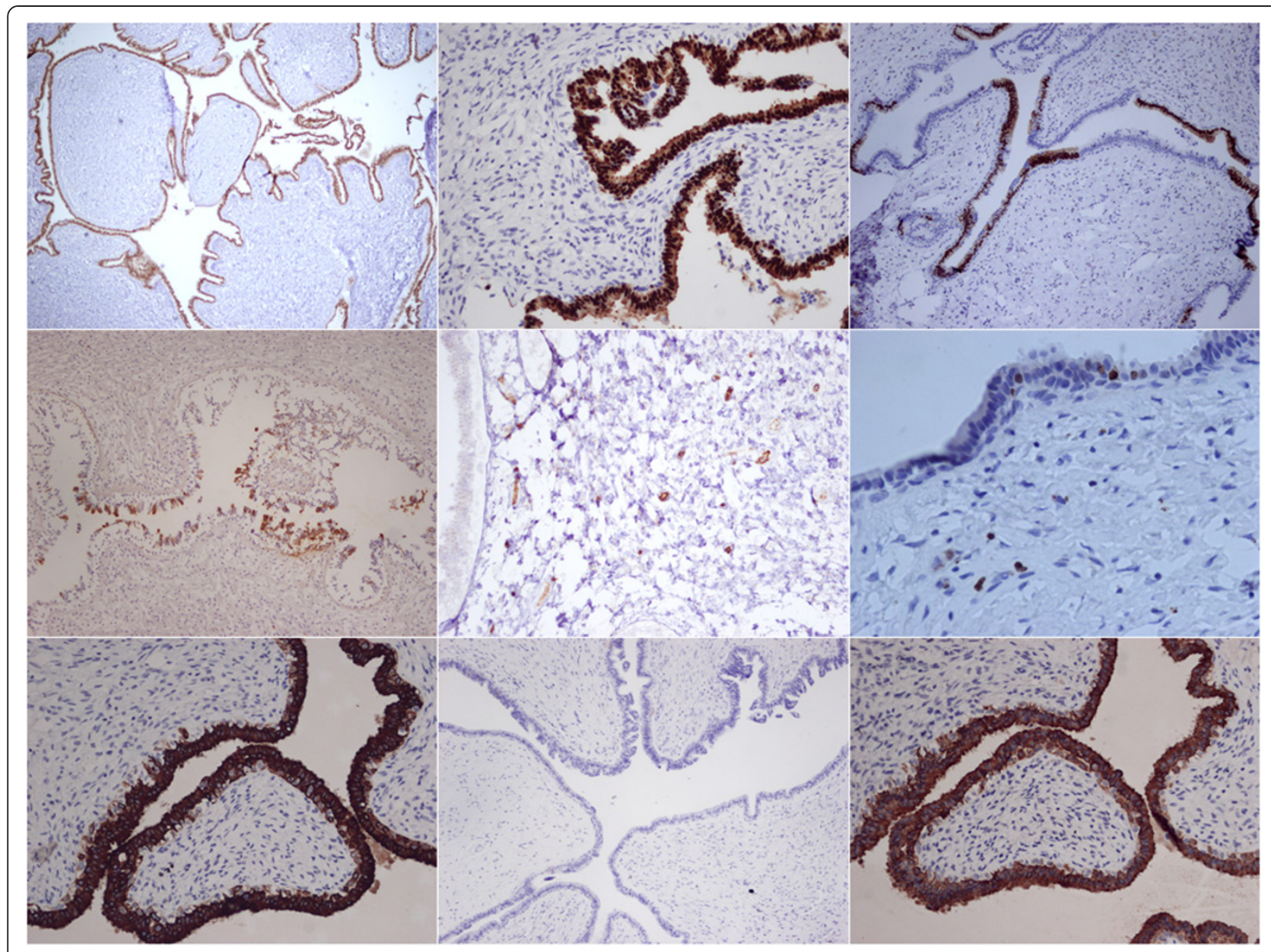

Figure 4 Immunohistochemistry. a) ER. b) PR. c) AR. d) GCDFP-15. e) CD34. f) Ki-67. g) CK7. h) PSAP. i) AE1/AE3.

elements. Cytokeratin stain can help visualize these tumor cells clearly (Figure 4i) [14]. Still another such tumor is fibroadenoma phyllodes, which is a histological feature similar to that of low-grade phyllodes tumor [15]. It can be difficult to distinguish the two because there is a continuum of morphologic findings. However, if the lesion has wellformed leaf-like slits and the hypocellular stroma appears to the degree that is found in fibroadenoma, it is called fibroadenoma phyllodes [15]. In our case, there was increased stromal cellularity and cell atypia. The proliferative index of stromal cells was found to be increased when viewing the Ki-67 Immunostain (Figure 4f). Together, these characteristics help distinguish phyllodes tumor from fibroadenoma phyllodes. Regardless, in the mammary gland, both fibroadenoma and phyllodes tumor carry risk of local recurrence.

Benign lesions in AGMLG outnumber malignant ones more than they do in the breast, possibly because the anus has more superficial locations [6]. In all patients reported previously, there was no recurrence of the tumor after excision [6]. As of twenty months follow-up, no local recurrence was found in our case. It is unclear whether phyllodes tumor of AGMLG has a better prognosis than phyllodes tumor of the mammary gland. Because it is difficult to predict behavior of this tumor in mammary glands, the possibility of recurrence locally cannot be totally excluded. Likewise, it would be prudent to closely follow-up phyllodes tumor of AGMLG postsurgery.

According to Sandra J. , there is always concurrent gynecomastia in fibroadenoma of breast in men [5]. Both gynecomastia and male breast cancer share hormonal imbalance as a same factor. Exogenous estrogen use in the male patient is evident [5]. Because our case had gynecomastoid hyperplasia and tumor on his AGMLG, we were concerned that he may also have a hormone imbalance as well as gynecomastia or tumor on the breast. However, his hormone levels (prolactin, betahCG, testosterone, E2, LH, FSH, T3, T4) were within normal range, and no gynecomastia or tumor was found in breast. These negative findings could be interpreted to suggest that our patient's phyllodes tumor and 
gynecomastoid hyperplasia of AGMLG resulted from localized hormone change. Although we found no peripheral hormone change, it might be still wise to monitor hormone levels in other such patients because of the known strong association between hormone level and gynecomastia as well as male breast cancer.

The alterations in ratio of androgen to estrogen may be influenced by many factors [16]. In middle-aged men, changes in this ratio can be caused by testicular or adrenal tumors, hormone-secreting tumors, hormone deficiency, prostate cancer, obesity, liver or renal disease, medical history of disease or drug abuse. Our male patient had normal hormone status (prolactin, beta-hCG, testosterone, E2, LH, FSH, T3, T4), and denied taking any drugs except for those he was taking for hypertension and depression. However, in such patients, it is necessary to take a careful medical and drug-use history as well as perform comprehensive evaluations, including physical examination, imaging, and laboratory evaluations.

\section{Conclusions}

In summary, herein we have introduced the first case of a male patient with a phyllodes tumor in anogenital mammary-like glands. Gynecomastoid hyperplasia of AGMLG was also identified, though no hormone imbalance or breast manifestations were found. We are unsure whether the phyllodes tumor of AGMLG lesion in our male patient was indeed a localized lesion or whether he would develop breast cancer in the future. Further study is needed to establish the clinical significance of this manifestation.

\section{Consent}

Written informed consent was obtained from the patient for publication of this case report and accompanying images. A copy of the written consent is available for review by the Editor-in-Chief of this journal.

\section{Abbreviations \\ AGMLG: Anogenital mammary-like glands.}

\section{Competing interests}

The authors declare that they have no competing interests.

\section{Authors' contributions}

HHT conceived the study, participated in its design, coordinated research efforts, and helped draft and edit the manuscript. SPH participated in the pathological analysis and drafted the manuscript. TMK and P-CC contributed to the collection of clinical data. All authors read and approved the final version of the manuscript.

\section{Author details}

'Department of Pathology and Laboratory Medicine, Kaohsiung Veterans General Hospital, Kaohsiung, Taiwan. ${ }^{2}$ Department of Colorectal Surgery, Kaohsiung Veterans General Hospital, Kaohsiung, Taiwan. ${ }^{3}$ Department of Psychiatry, Kaohsiung Veterans General Hospital, Kaohsiung, Taiwan.

\section{References}

1. Van der Putte SC: Mammary-like glands of the vulva and their disorders. Int J Gynecol Pathol 1994, 13:150-160.

2. Kazakov DV, Spagnolo DV, Stewart CJ, Thompson J, Agaimy A, Magro G, Bisceglia M, Vazmitel M, Kacerovska D, Kutzner H, Mukensnabl P, Michal M: Fibroadenoma and phyllodes tumors of anogenital mammary-like glands: a series of 13 neoplasms in 12 cases, including mammary-type juvenile fibroadenoma, fibroadenoma with lactation changes, and neurofibromatosis-associated pseudoangiomatous stromal hyperplasia with multinucleated giant cells. Am J Surg Pathol 2010, 34(1):95-103.

3. Doganavsargil B, Akalin T, YImaz M, Kandiloglu G: Perianal fibroadenoma, case report. Am J Dermatopathol 2008, 30(1):81-3.

4. Choi WW, Tadros TS, Majmudar B: Anal fibroadenoma: report of a common tumor type in an unusual location. South Med J 2007, 100(9):914-5.

5. Shin SJ, Rosen PP: Bilateral presentation of fibroadenoma with digital fibroma-like inclusions in the male breast. Arch Pathol Lab Med 2007, 131(7):1126-9.

6. Kazakov DV, Spagnolo DV, Kacerovska D, Michal M: Lesions of anogenital mammary-like glands: an update. Adv Anat Pathol 2011, 18(1):1-28.

7. Scurry J, van der Putte SC, Pyman J, Chetty N, Szabo R: Mammary-like gland adenoma of the vulva: review of 46 cases. Pathology 2009, 41(4):372-8.

8. Hatanaka K, Tanimoto A, Umekita Y, Yoshioka T, Kanekura T: Unusual anogenital apocrine tumor resembling mammary-like gland adenoma in male perineum: a case report. Diagn Pathol 2010, 5:42.

9. Chih-Yi L, Shen M-H, Yih-Yiing W: Unusual Cystic Apocrine Adenoma in the Male Anus: A Case Report and Review of the Literature. FJJM 2011, 9(3):151-156.

10. McNiff J, McCalmont TH, Requena L, Sangüeza OP, Vassallo C, Rosso R, Borroni G, Glusac EJ, Pichardo RO: Benign tumours with apocrine and eccrine differentiation. In Pathology And Genetics of Skin Tumours. Edited by LeBoit PE, Burg G, Weedon D, Sarasin A. IARC Press: Lyon; 2006:147 [World Health Organization Classification of Tumours].

11. Kazakov DV, Stewart CJ, Kacerovska D, Leake R, Kreuzberg B, Chudacek Z, Hora M, Michal M: Prostatic-type tissue in the lower female genital tract: a morphologic spectrum, including vaginal tubulosquamous polyp, adenomyomatous hyperplasia of paraurethral Skene glands (femaleprostate), and ectopic lesion in the vulva. Am J Surg Pathol 2010, 34(7):950-5

12. Nucci MR, Ferry JA, Young RH: Ectopic prostatic tissue in the uterine cervix: a report of four cases and review of ectopicprostatic tissue. Am $J$ Surg Pathol 2000, 24(9):1224-30.

13. Tekin K, Sungurtekin U, Aytekin FO, Calli N, Erdem E, Ozden A, Yalçin N: Ectopic prostatic tissue of the anal canal presenting with rectal bleeding: report of a case. Dis Colon Rectum 2002, 45(7):979-80.

14. Hicks DG, Lester SC: Fibroadenoma and phyllodes tumor. In Diagnostic Pathology: Breast. Edited by Ashley R, Renlund MA, Arthur G, Gelsinger MA, Matthew R, Connelly MA, Lorna Morring MS, Alicia M, Moulton BA, Rebecca L, Hutchinson BA, Angela M, Green BA, Jeffrey J, Marmorstone BS, Lisa A, Magar BS, Dora M, Lam-Himlin MD, Nicole Winkler MD. Salt Lake City, UT: AIRSYS ${ }^{\circledR}$ Press; 2011:6:5-6:13.

15. Tavassoli F, Eusebi V: Biphasic Tumor. In Tumors of the mammary gland. Edited by Silverberg SG. Washington, DC: American Registry of Pathology, Armed Forces Institute of Pathology Press; 2009:320-325. AFIP Atlas of tumor Pathology, series 4, fascicle 10.

16. Matsumoto AM, Bremner WJ: Testicular Disorders. In Williams textbook of endocrinology. 12th edition. Edited by Melmed S, Polonsky KS, Larsen PR, Kronenberg HM. Philadelphia, PA: Saunders/Elsevier Press; 2011:718-721.

doi:10.1186/1746-1596-8-49

Cite this article as: Ho et al:: Anal phyllodes tumor in a male patient: a unique case presentation and literature review. Diagnostic Pathology 2013 8:49. 